\title{
INTERCAMBIADOR DE TRANSPORTES DE LA PLAZA DE CASTILLA
}

\section{( PUBLIC TRANSPORT INTERCHANGE AT THE PLAZA DE CASTILLA )}

Tomás Dominguez del Castillo y Juan Carlos Martín Baranda

Fecha de recepción: 6-II-95

Arquitectos

\section{RESUMEN}

En el presente articulo se exponen las principales caracteristicas de la obra del Intercambiador de Transportes de la Plaza de Castilla de Madrid, basada en criterios estéticos y funcionales adecuados a su finalidad y se comentan detalladamente sus usos y datos constructivos.
SUMMARY

This article describes the main characteristcs of the Public Transport Interchange at the Plaza de Castilla in Madrid, based on the aesthetic and functional criteria adapted to the purpose of the work. It also contains detailed description of the use and construction data.

\section{Antecedentes}

El Ayuntamiento de Madrid convocó el Concurso de Ideas "Intercambiador Plaza de Castilla" con el objeto de seleccionar la propuesta que mejor resolviera el doble reto de:

1.- Construir un Intercambiador de Transportes, que constituía en sí mismo un objetivo intrínseco de carácter funcional, derivado de los compromisos del Convenio Urbanístico "Plaza de Castilla".

2.- Diseñar una solución presidida por las condiciones especiales de adaptación al entorno de la plaza de Castilla en la que se ubica.

Las dificultades derivadas de este doble reto residían, en lo que se refiere al propio Intercambiador, en el elevado número de viajeros que utilizan los transportes públicos en la zona, estimado en 260.000 viajeros/día.

En lo referente a su integración en el entorno, la existencia de un gran túnel viario y una doble galería de servicios en el subsuelo en que se asienta y la presencia próxima de las torres inclinadas de Puerta de Europa por encima del conjunto, con su espectacular diseño, hacían difícil la consecución de una propuesta armónica con dicho entorno.
A esta dificultad se añadía la propia configuración de la plaza de Castilla, formada por varios elementos singulares (monumento a Calvo Sotelo, fuente central, así como el resto de edificios de la plaza, incluido el depósito del Canal de Isabel II).

En julio de 1992 se produjo el fallo del Concurso de Ideas, que recayó en la propuesta de loa arquitectos Tomás Domínguez del Castillo y Juan Carlos Martín Baranda, tras reñida competición con las soluciones de los más destacados proyectistas. La solución ganadora destacaba por "constituir un conjunto formal y constructivamente ligero, considerando la diversidad de elementos que se alinean en el eje de la Castellana".

En marzo de 1993, el Consejo de Gerencia Municipal de Urbanismo aprobó el proyecto redactado por los arquitectos ganadores del Concurso.

La Empresa Proyectos y Desarrollos Urbanísticos y Financieros, S.A. (PRODUSA), asumió los compromisos anteriormente establecidos por URBANOR, S.A. en el Convenio Urbanístico Plaza de Castilla y adjudicó las obras correspondientes a FOMENTO DE CONSTRUCCIONES Y CONTRATAS, S.A., bajo la dirección del Ayuntamiento de Madrid. 
El Intercambiador fue inaugurado por el Excmo. Sr. Alcalde de Madrid en diciembre de 1994.

\section{Descripción}

La propuesta incluye un único edificio semicerrado en el área situada más al Norte, que alberga el programa de servicios que necesitan una mayor protección.

El límite de actuación en superficie viene configurado de la siguiente forma:

- NORTE - Alineación con el bordillo de la acera del Sur de la calle San Benito.

- ESTE - Alineación con el bordillo de la acera del paseo de la Castellana, sin incluir dicha acera.

- SUR - Alineación que incluirá la isleta curva que ayuda a completar el círculo de la plaza de Castilla.

- OESTE - Alineación con el bordillo de la acera del paseo de la Castellana, sin incluir dicha acera.

La propuesta incluye:

- 13 dársenas para viajeros, con una capacidad de autobuses por dársena, de 2 , de tipo articulado ó 3 de dos ejes.

- 7 carriles sencillos para autobuses

- 5 carriles dobles para autobuses

- 104 unidades de parasoles

- torre de 18,40 metros de altura

- zona de parada de taxis en las dársenas norte y sur.

- servicio de atención al viajero formado por taquillas, oficinas, cafetería, área de espera cubierta, aseos, teléfonos y áreas de anuncios.

- mobiliario urbano en las dársenas (bancos y papeleras).

\section{1.- Criterios formales}

A partir de la idea básica de que la ligereza debe presidir todo el conjunto, se emplea un elemento repetitivo de gran esbeltez, el paraguas-sombrilla, con una dimensión en altura de siete metros.

El primer tramo, con una altura de 4,5 metros y cubierto por un entramado, forma techo continuo transparente, que hace el efecto de paraguas y que va apoyado en una hilera de pilares que configuran el andén.
El segundotramo, hasta los siete metros, se forma agrupando cuatro placas, perforadas alrededor de cada pilar, dando lugar a las sombrillas. Por la disposición de los andenes, la orientación Norte-Sur proporciona sol en inviernoy sombra en verano, afectando eí sol de verano sólo a los extremos de los andenes.

En la primera dársena y más al Norte se van a desarrollar los usos cerrados más solicitados a los cuales se les da un aspecto ligero, de contenedores protegidos sobre cubierta por los elementos paraguas-sombrilla. Este planteamiento permitirá afrontar unas sobrecargas muy ligeras, por debajo de lo admisible, teniendo en cuenta la servidumbre de servicios que hay bajo rasante (túnel de tráfico privado y público, túnel de metro a cocheras, galerías de servicio, etc.).

\section{2.- Descripción de usos}

\section{Dársena 1}

Es la situada más al Norte y, junto con la última, es la de mayor superficie $(50 \times 18,20 \mathrm{~m})$. Sobre ella se implantan los usos de atención al viajero que se detallan:

a.- Dos edificios para venta de billetes y oficinas de las compañías adjudicatarias de líneas, formando un conjunto de cinco cabinas sencillas, dos cabinas dobles y una cabina para la E.M.T (Empresa Municipal de Transportes)., a espaldas de las cuales existen dos áreas abiertas para oficinas.

A cada uno de los dos edificios se les da la posibilidad de dos salidas, para que cada uno de ellos se pueda compartimentar en dos espacios independientes.

b.- Un aseo de hombres compuesto por cuatro lavabos, dos cabinas para inodoros, con fluxómetro y tres urinarios.

Un aseo de mujeres compuesto por cuatro lavabos y cuatro cabinas de inodoros, con fluxómetro.

c.- Bar-Cafetería. Situada a la sombra de la torre metálica, consta de una barra circular, con un espacio a sus espaldas destinado a almacenamiento. Su funcionamiento es el de un kiosco, con la diferencia de que, en este caso, el área de clientes está protegida de la lluvia y el sol por las marquesinas que cubren toda la dársena.Los efectos de tipo horizontal producidos por otras inclemencias del tiempo (viento, lluvia con viento, etc.) se solucionan cerrando la cara norte con mamparas de vidrio que se extienden desde el pavimento hasta las marquesinas.

d.- Zona de espera. Se sitúa entre el borde norte y el barcafetería. Este área está cubierta y protegida lateralmente. Sin embargo, no es un área cerrada totalmente y sobre ella existe una serie de elementos de mobiliariourbano(bancos- 


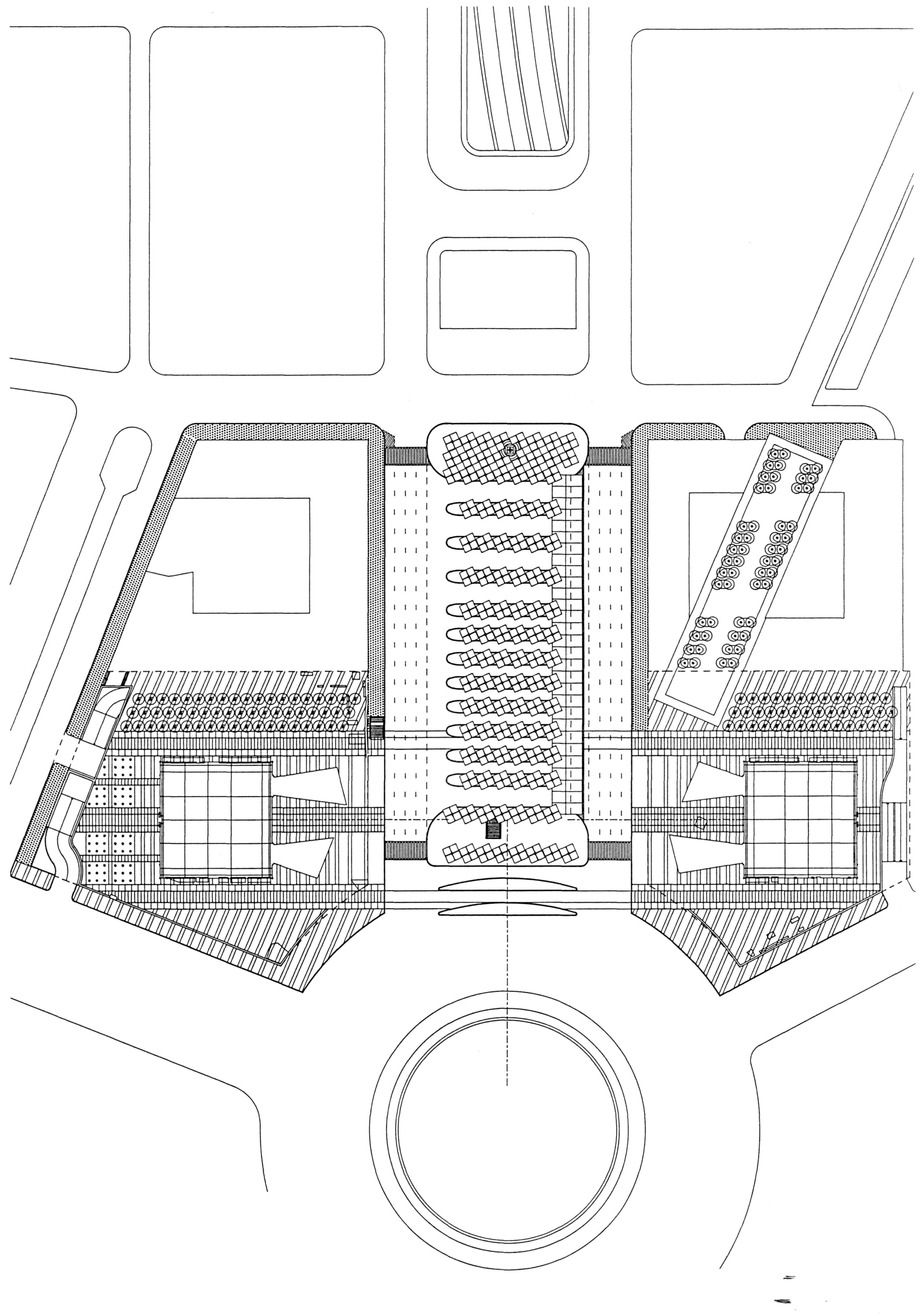


38

de la Construcción, Vol. 46 n ํ 436, marzo/abril 1995

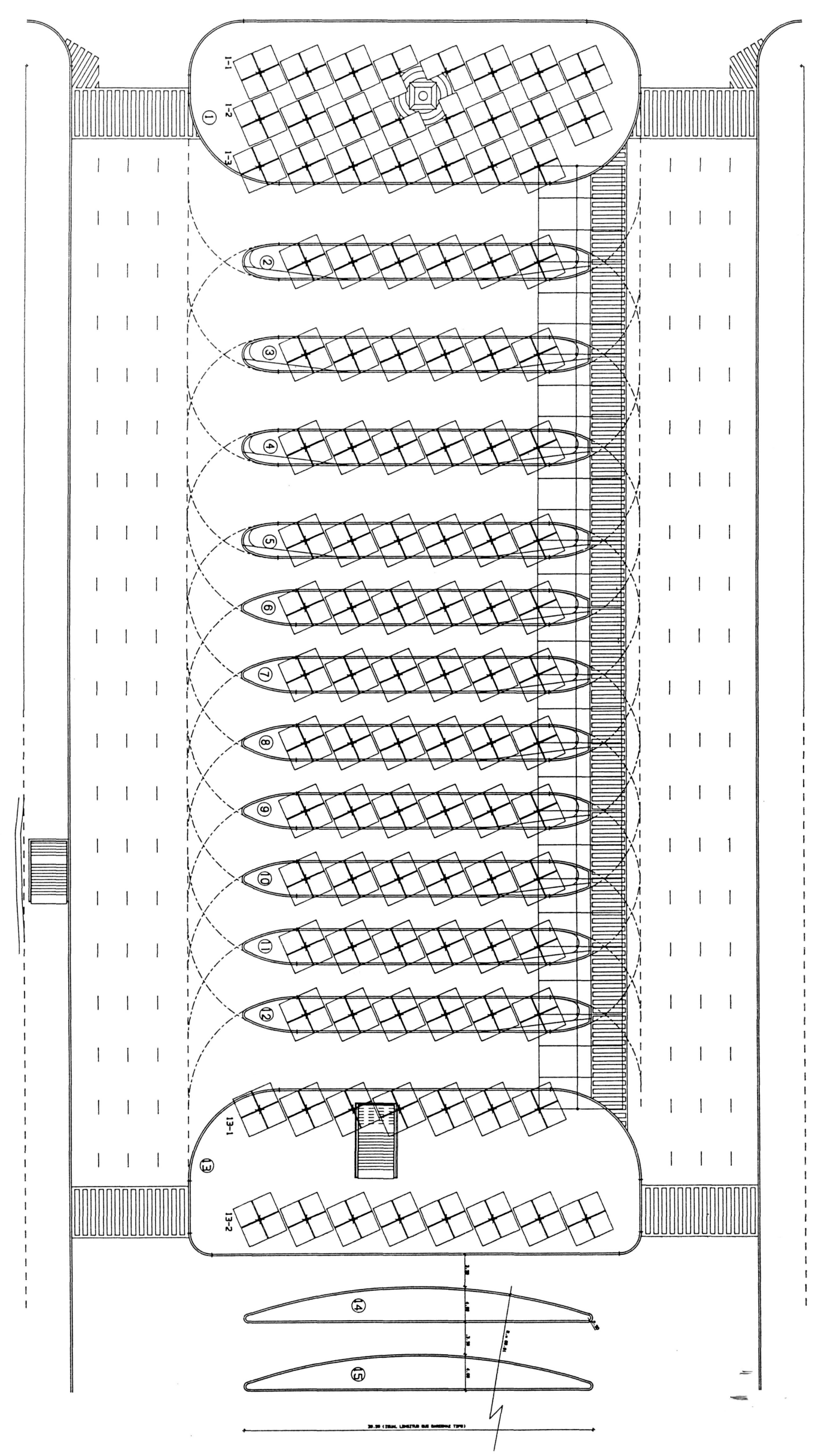

(c) Consejo Superior de Investigaciones Científicas
Licencia Creative Commons 3.0 España (by-nc)

http://informesdelaconstruccion.revistas.csic.es 
mesas, ceniceros, etc), todos ellos atornillados al suelo para mantener el área ordenada.

e.- Áreas de teléfonos situados a ambos lados de la zona de espera y compuestas cada una de ellas de dos teléfonos, cuatro en total. Esta dotación se podría aumentar en función de los requerimientos.

Todos estos elementos independientes se cobijan debajo de las marquesinas que mantienen una modulación igual a lo largo de todo el Intercambiador. Esto permitirá en el futuro organizar implantaciones de cualquier tipo en función de otras necesidades y dentro de los límites de que se dispone y de las sobrecargas admisibles.
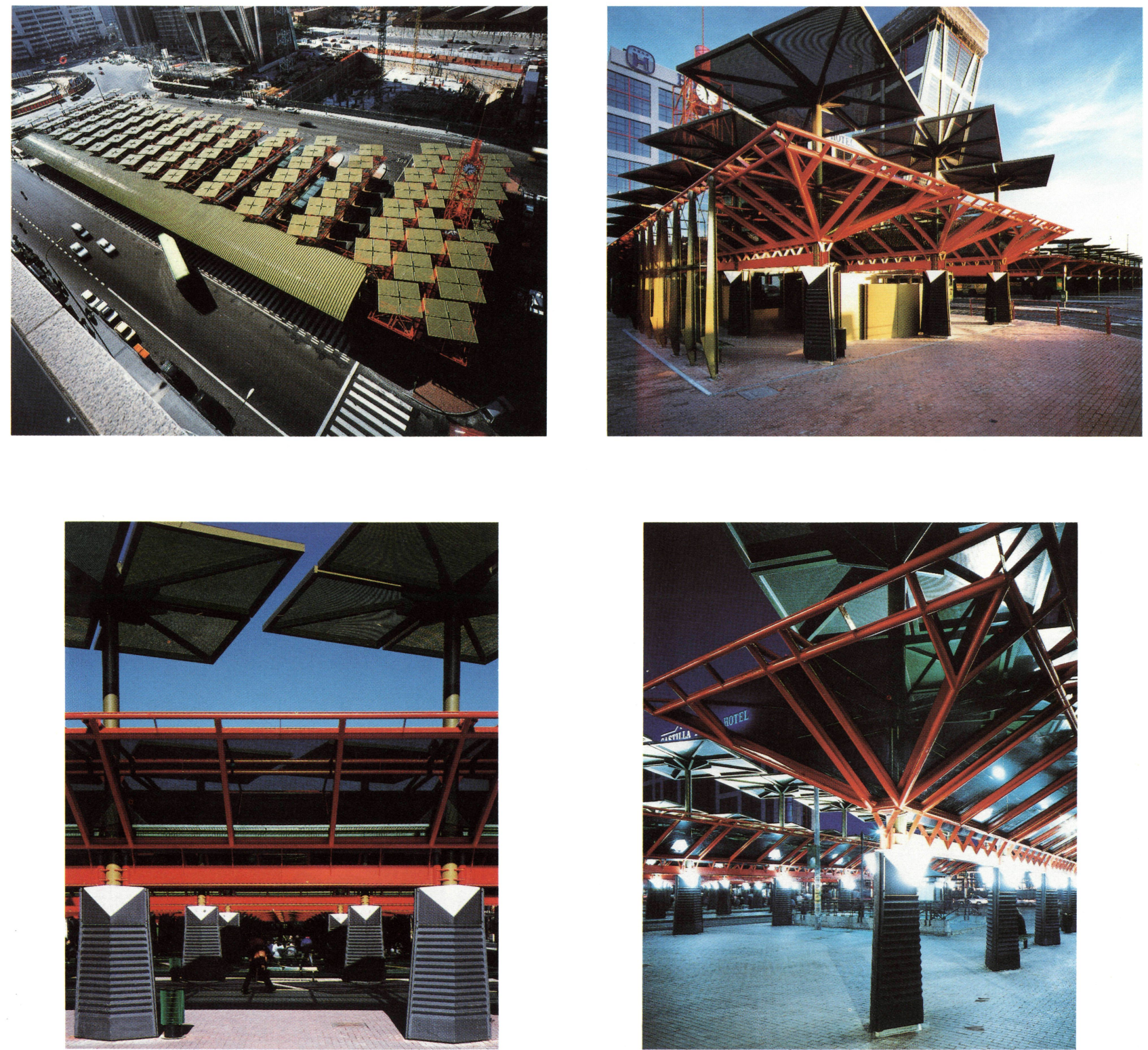


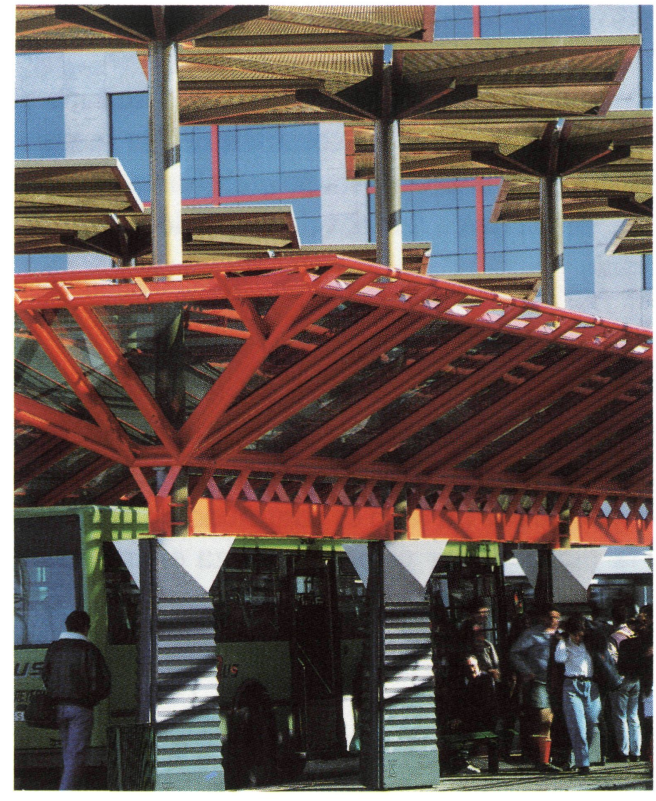

autobuses de líneas del exterior. Asimismo se encuentra allí la boca más próxima a la estación de Metro. Por estas razones, el área de atención al viajero está ubicada en el lado opuesto, repartiendo de esta manera los movimientos peatonales que se producen.

\section{Torre Metálica}

Tienelafunción de llamada o indicador del Intercambiador, un elemento que destacará dentro de la horizontalidad del conjunto. Elemento reclamo sobre el que se pueden colocar nombres o anagramas, viene rematado por un reloj de cuatro esferas, una en cada cara.

Toda la torre es metálica, formada con un tubo de acero redondo, de $80 \mathrm{~mm}$ de diámetro y de $3 \mathrm{~mm}$ de espesor, que le dará un aspecto de gran ligereza desde el punto de vista formal y estructural.
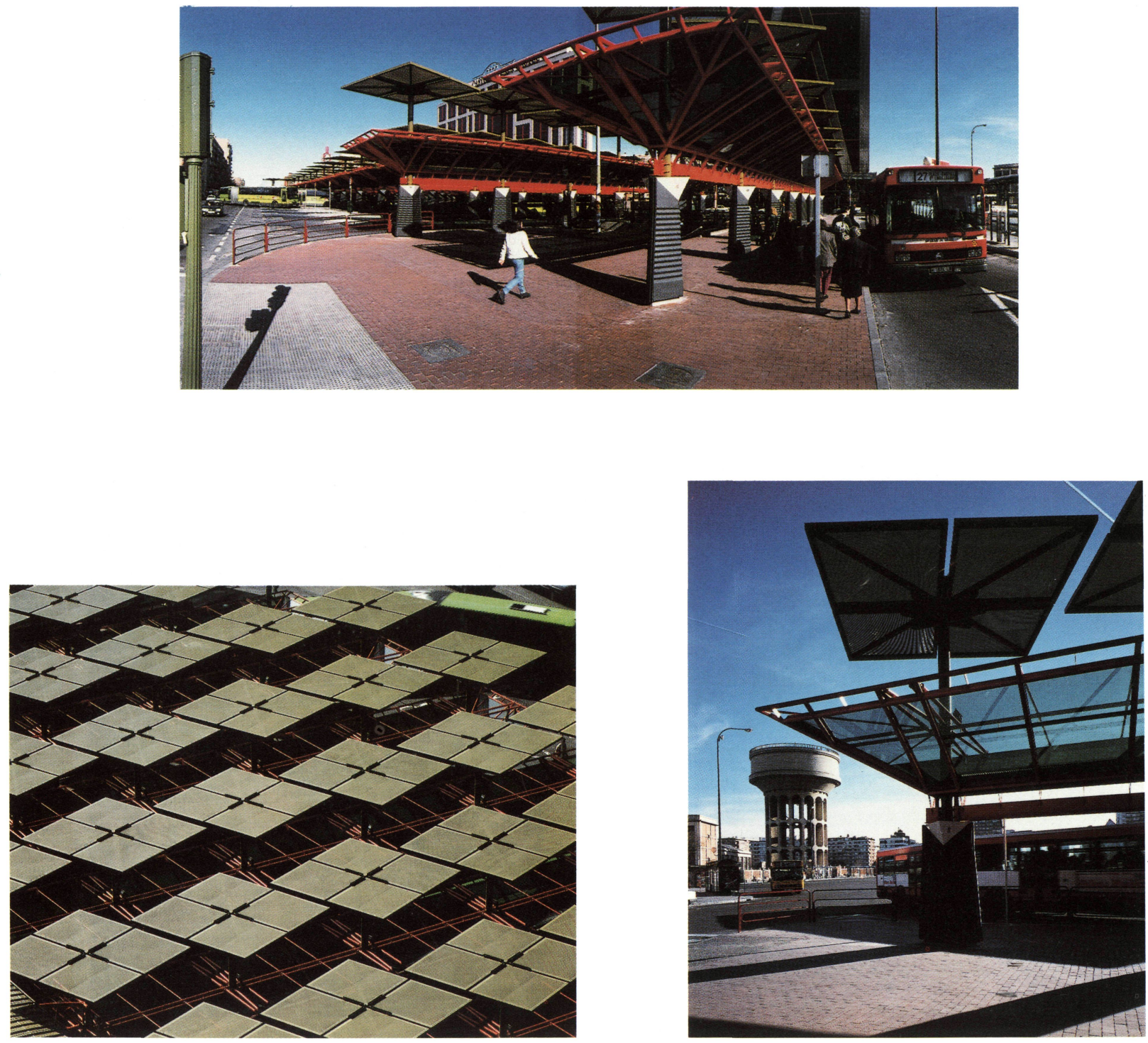

(c) Consejo Superior de Investigaciones Científicas 


\section{3.- Elementos constructivos}

\section{Paraguas-Sombrilla}

Elemento repetitivo, formado por un pilar metálico de acero, de $250 \mathrm{~mm}$ de diámetro y $20 \mathrm{~mm}$ de espesor, del que nace, en su punto intermedio, una marquesina a dos aguas, transparente.

Se corona el pilar a una altura de siete metros con una sombrilla cuadrada subdividida en cuatro cuadrados y que está formada por una estructura tubular de sección $100 \times 100 \times 4$, cubierta con chapa perforada de $2 \mathrm{~mm}$.

Desde el arranque del pilar hasta una altura de 2,40 metros y para proteger las bajantes, se colocan dos piezas desmontables de hierro fundido, estriados horizontalmente para evitar que se peguen carteles, así como para rigidizar más estas piezas.

\section{Oficinas-Taquillas}

Sobre un forjado metálico con perfiles IPN140 se forma una cubierta de chapa ligera a dos aguas dentro de las cuales se ubica el aire acondicionado. Un falso techo metálico-registrable modulado a $120 \times 30$ cierra el techo.

Las divisiones interiores son mamparas de lunas securizadas de $10 \mathrm{~mm}$, ancladas a suelo y techo con elementos de acero inoxidable (accesorios normalizados). El cerramiento exterior es de climalit, siendo la luna exterior ST ADIP de $8+8$, cámara de 12 y luna interior securizada de $10 \mathrm{~mm}$

Este cerramiento se resuelve horizontalmente con zócalo de acero inoxidable corrido, anclado al suelo y, en la parte superior, con un perfil horizontal en $\mathrm{L}$, anclado al forjado metálico de junquillos de cuadradillo de acero inoxidable.

Las uniones verticales entre lunas se realizan a base de siliconas y con maineles verticales de luna de $10 \mathrm{~mm}$

El pavimento es de goma tipo Pirelli o similar. Todo el conjunto disfrutará de gran diafanidad, protegiéndose internamente de la visión, si se desea, mediante persianas ligeras o estores.

\section{Aseos}

Los aseos se configuran con cuatro elementos de hormigón prefabricado, para pintarlos posteriormente. Estos cuatro elementos cierran las cuatro caras verticales, dos de ellas compuestas en forma de $\mathrm{U}$ invertida para albergar las cabinas y dos muros, uno recto, que se inclina para dar paso a la puerta que cierra el tercer lado y un muro que envuelve el resto del conjunto. El área donde se ubican los lavabos queda abierta protegiéndose con una estructura metálica para sustentar una pérgola de mallazo que termina de cerrar el conjunto
Bar-Cafetería

Forma un volumen de base poligonal y barra circular en un $75 \%$, quedando el otro $25 \%$ para almacenamiento.

El bar-cafetería se cierra cuando está fuera de servicio con persianas enrollables metálicas de seguridad, que se ocultan en el grueso de la marquesina.

\section{Torre Metálica}

De base cuadrada de $2 \times 2$ metros y cuatro aletas que salen de sus diagonales, arranca hacia arriba en tramos de 2 metros de alto, hasta un total de 6 tramos, coronados con un cilindro hueco de chapa perforada y rematado en una antena. Toda la estructura es tubular.

En el último tramo de dos metros hay cuatro esferas de reloj de $1,20 \mathrm{~m}$ de diámetro.

El conjunto se asienta sobre cuatro pilares de $250 \mathrm{~mm}$ de diámetro que atraviesan el bar estratégicamente.

\section{Paso de Peatones}

La comunicación norte-sur entre dársenas se establece mediante un paso de peatones de 5 metros de ancho, señalizado por el cebreado correspondiente, asi como las bandas sonoras de borde. Todo ello va cubierto por una marquesina longitudinal volada sobre el paso, lo que permite el tránsito peatonal protegido de la lluvia, configurando además un espacio de comunicación perfectamente delimitadoe integrador de todas las dársenas

\section{Pavimentación}

\section{Calzada}

Se utiliza un firme para tráfico pesado compuesto de

- mezcla bituminosa en caliente s/art. 40.63 del pctg,

- base de hormigón tipo H-125 s/art. 40.41 del pctg,

- arena de miga s/art. 40.41 del pctg, y

- suelo adecuado compactado al $100 \%$ del proctor normal s/art. 40.16 del pctg.

\section{Bordillo}

El bordillo, de las dimensiones establecidas por el Ayuntamiento de Madrid para delimitar aceras de calzadas, está compuesto por:

- adoquín cerámico de clínker, de $200 \overline{\mathrm{x}} 100$ x 4,5

- arena seleccionada

- base de arena de miga. 


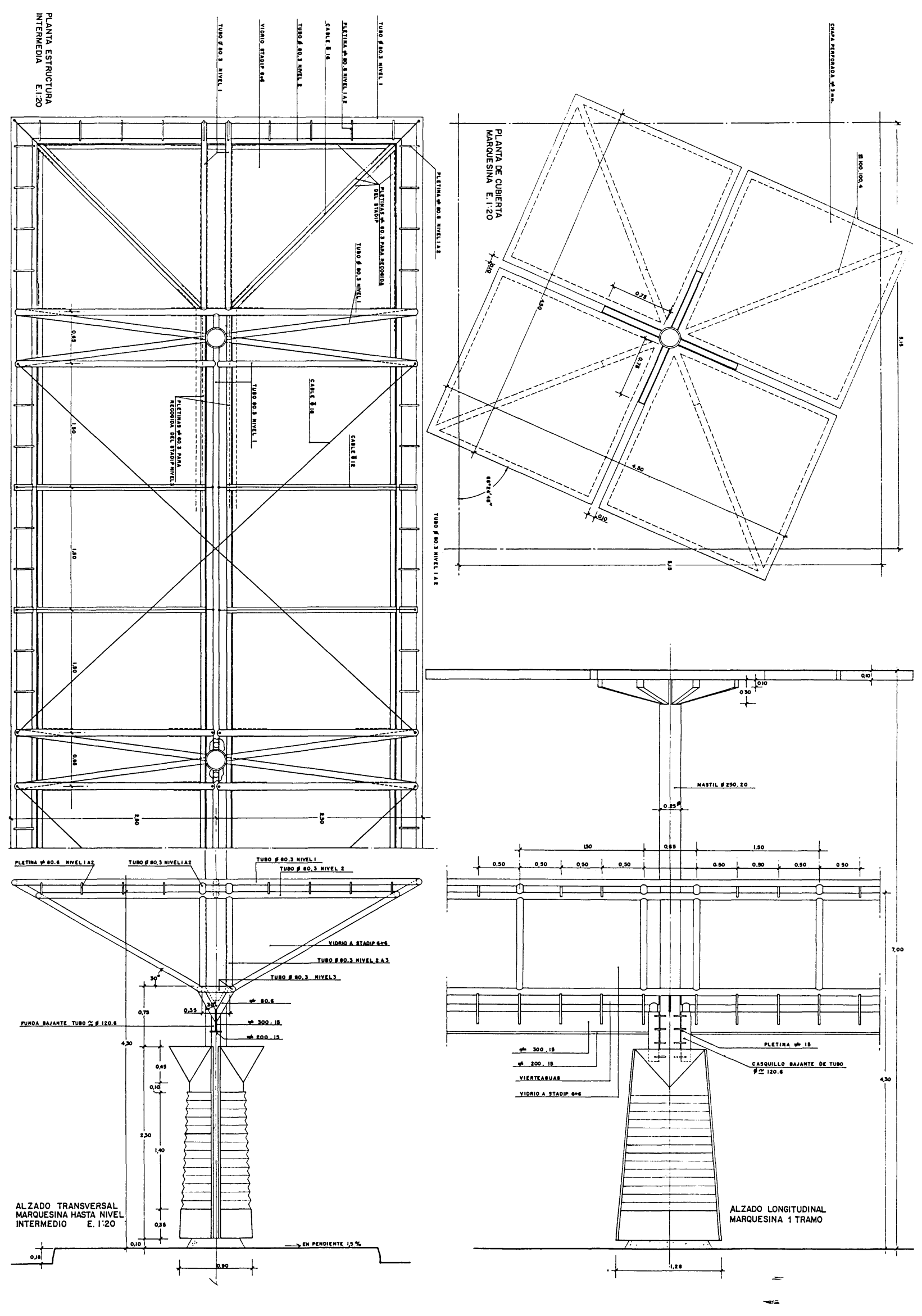



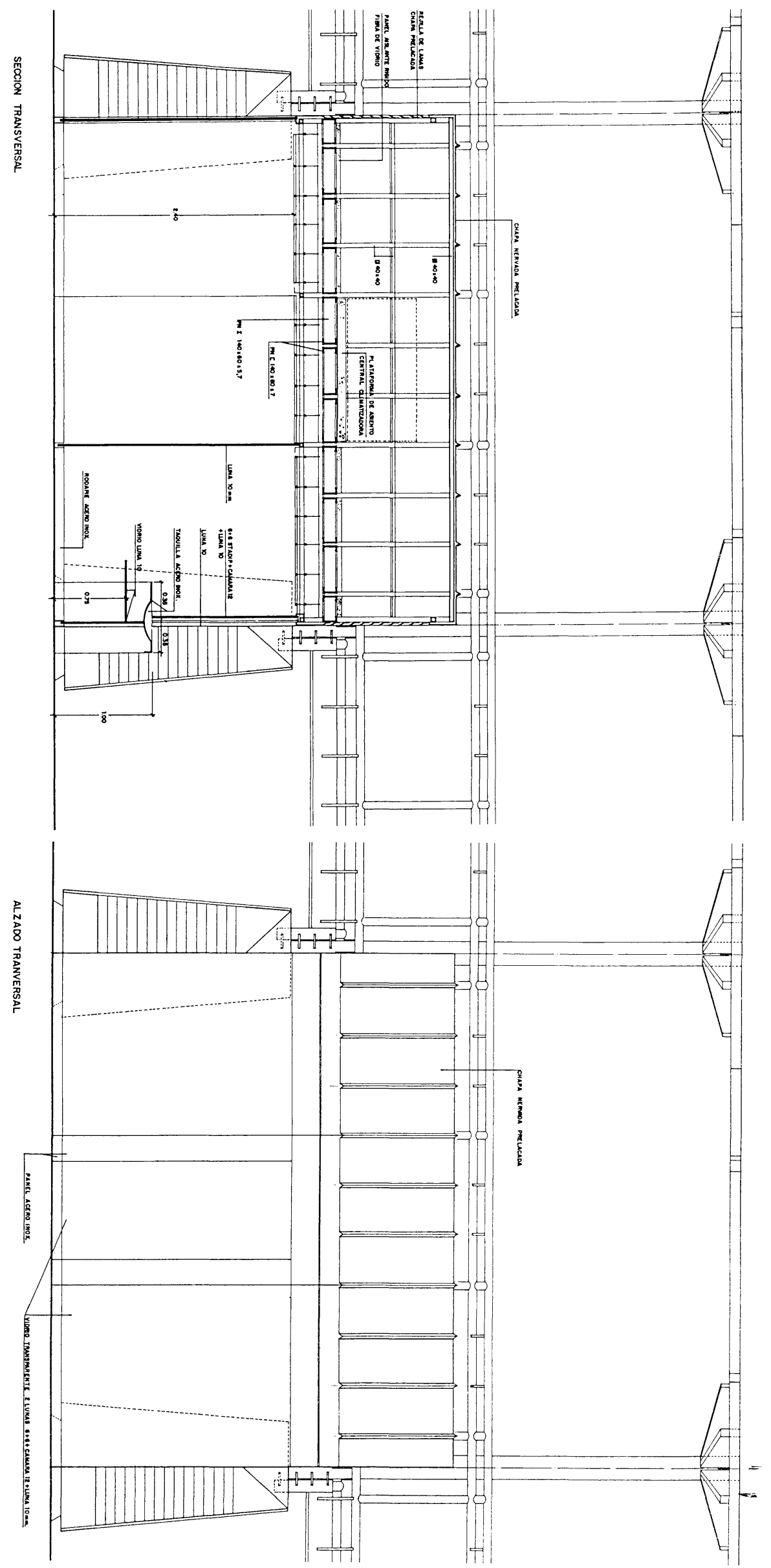


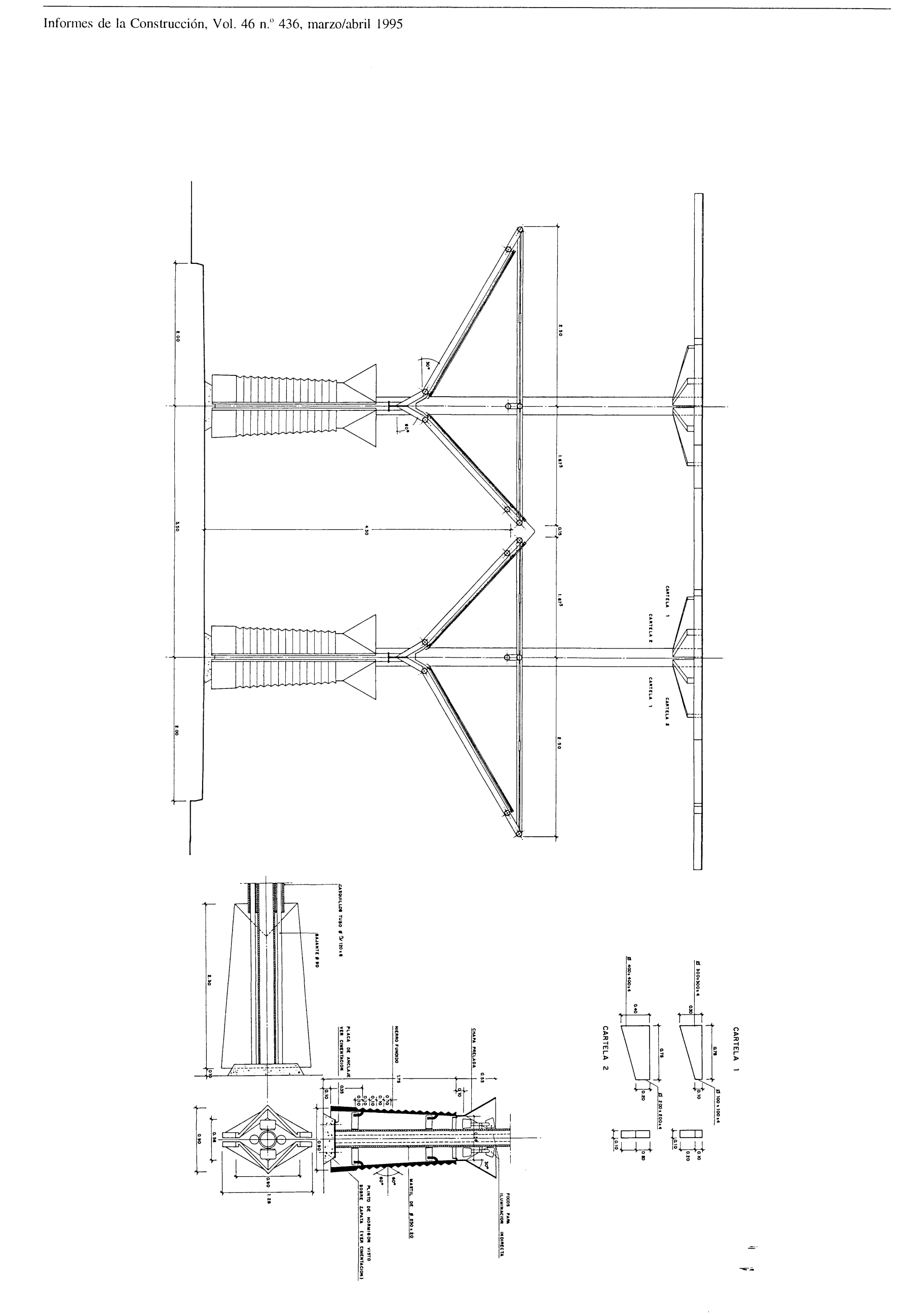



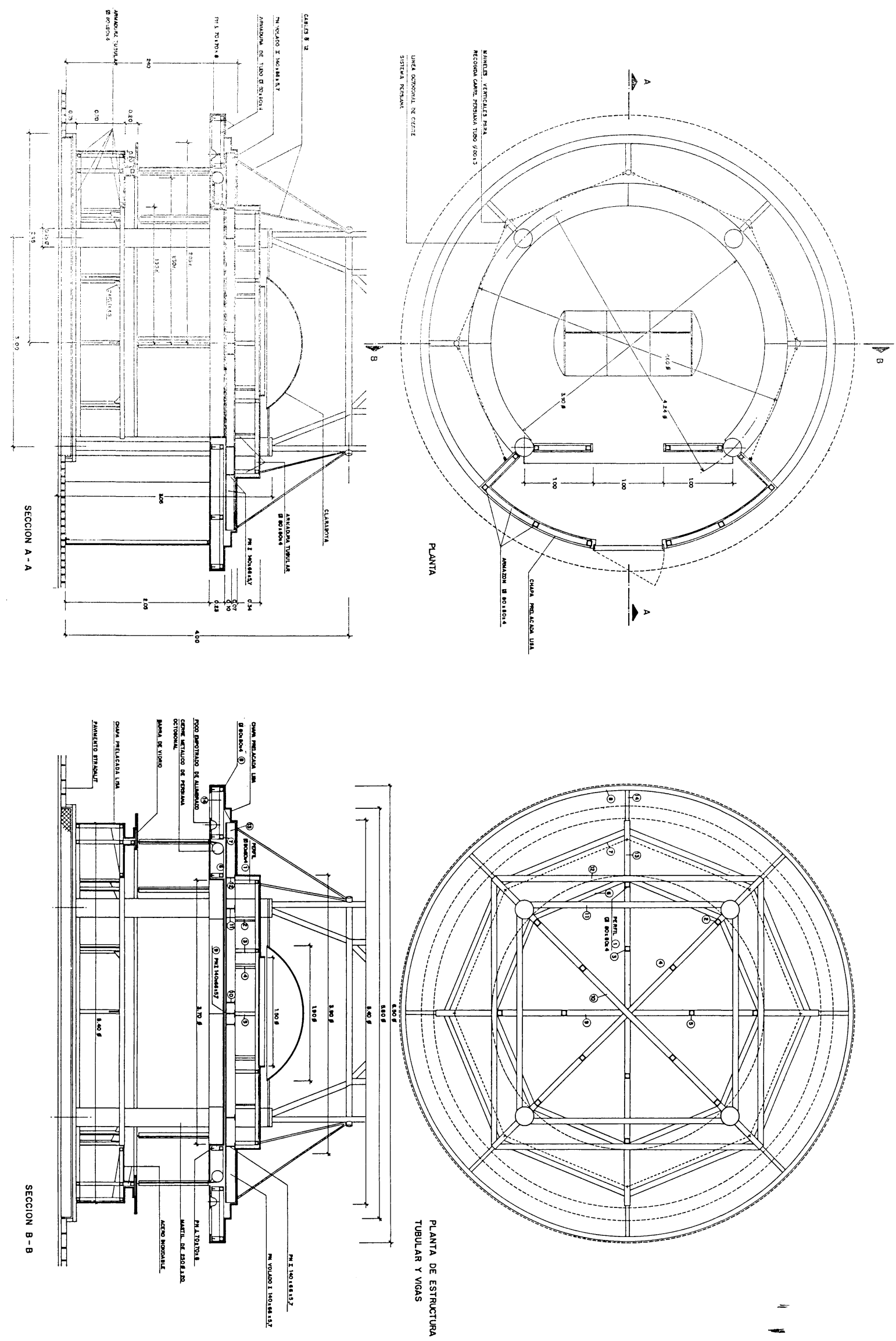


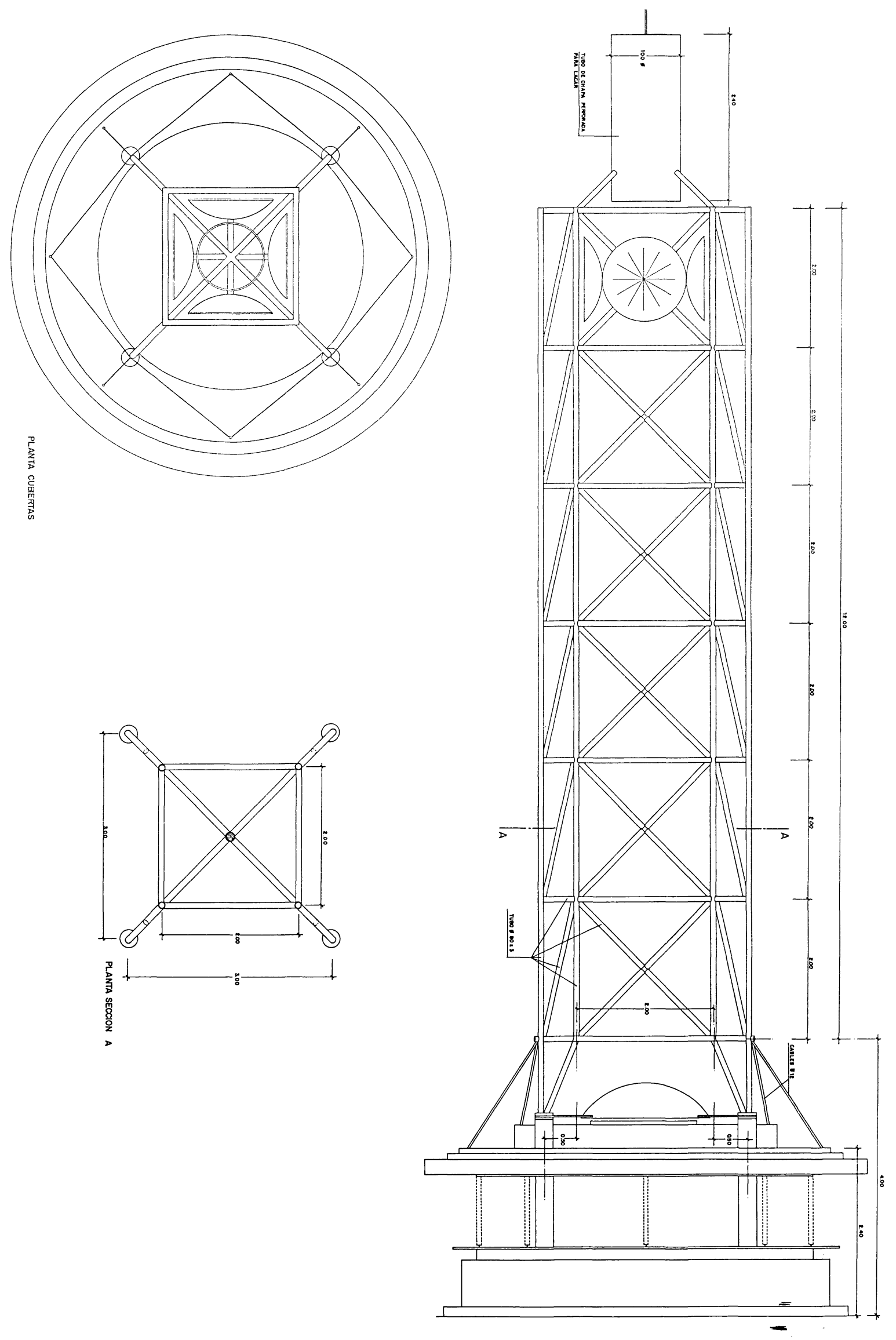


\section{Ficha técnica}

Propiedad: $\quad$ Excmo. Ayuntamiento de Madrid

Autores del Proyecto: D. Tomás Domínguez del Castillo y D. Juan Carlos Martín Baranda, Arquitectos.

Dirección del Proyecto: D. Ricardo Castro Canseco. Ingeniero de Caminos, Canales y Puertos.

Departamento de Proyectos de Urbanización Sistemas locales. Ayuntamiento de Madrid. Arquitecto Jefe D. Arturo

Ordozgoiti Blázquez.

Dirección de las Obras: D. Ricardo Castro Canseco. Ingeniero de Caminos, Canales y Puertos.

D. Filemón Fernández Rodríguez Ingeniero T. de Obras Públicas.

Departamento de Proyectos de Urbanización Sistemas Locales. Ayuntamiento de Madrid. Arquitecto Jefe D. Arturo Ordozgoiti Blázquez

Construcción: $\quad$ PRODUSA. D. Juan Cervigón Simó

Contrata: FOMENTO DE CONSTRUCCIONES Y CONTRATAS, S.A. D. Emilio Bargueño

Control de las obras: EPTISA

Control de calidad: INTEMAC

Estructura Metálica: F. MONOPLEX, S.A.

Pintura: S.A. JULIO CRESPO

Electricidad: ESP. ELÉCTRICAS, S.A. (ESPELSA)

Chapa y Aluminio: FERDA, S.A.

Suministro Adoquín: CERAM. MALPESA

Suministro P. Fundición: FUNDICIÓN MACEDA

Metacrilato: PLEXI, S.A. GRUPO ROHM

Colocación Adoquín: BENITO AGRA E HIJOS

Colocación Metacrilato: CUB. CELULARES, S.A.

\section{Publicación del Instituto Eduardo Torroja-CSIC}

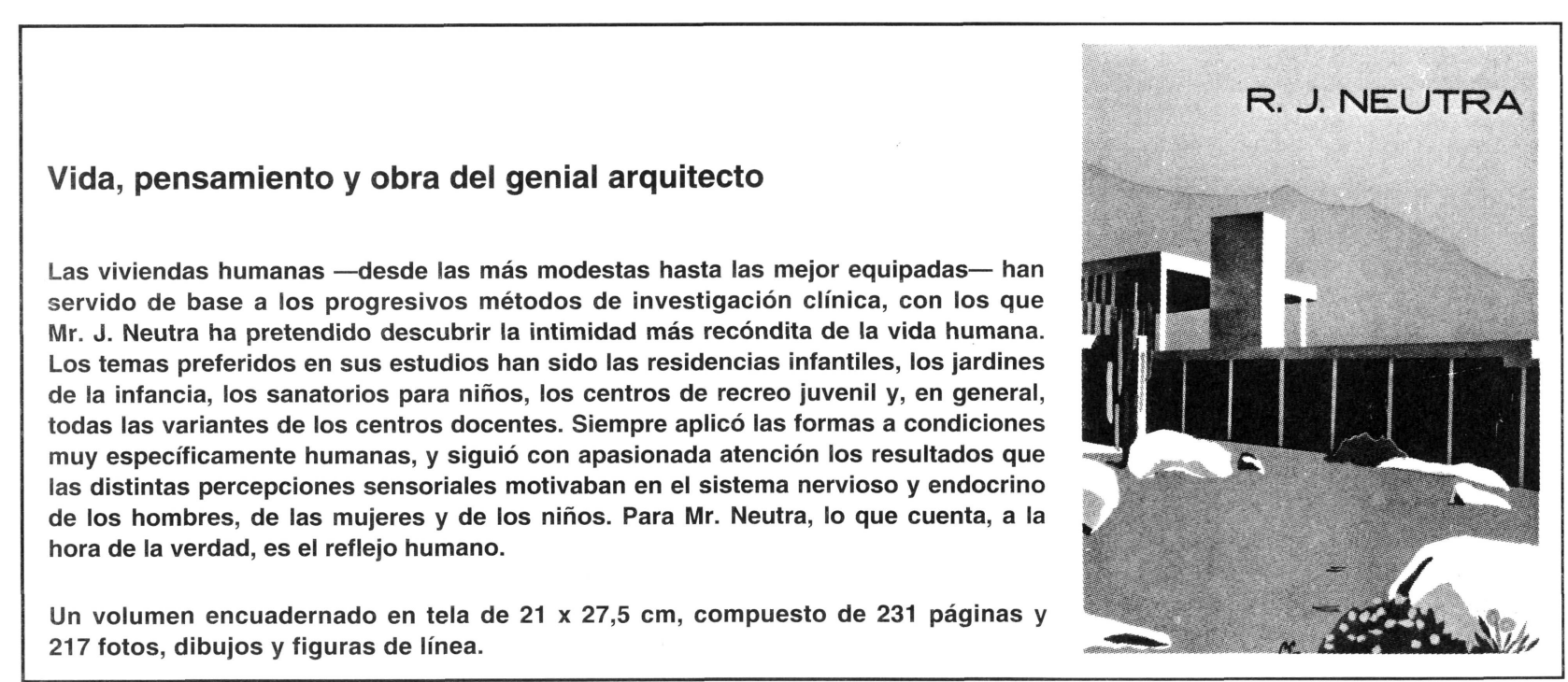

